\title{
Downregulation of CD9 promotes pancreatic cancer growth and metastasis through upregulation of epidermal growth factor on the cell surface
}

\author{
MAOCHUN TANG, GUOJIAN YIN, FENG WANG, HUA LIU, SHU ZHOU, \\ JIANBO NI, CONGYING CHEN, YINGQUN ZHOU and YAN ZHAO
}

Department of Gastroenterology, Shanghai Tenth People's Hospital, Tongji University School of Medicine, Shanghai 200072, P.R. China

Received January 30, 2015; Accepted April 21, 2015

DOI: 10.3892/or.2015.3960

\begin{abstract}
The expression of CD9 has been shown to be inversely associated with pancreatic cancer metastasis but the underlying mechanism remains incompletely understood. Using the two closely associated pancreatic cancer cell lines, PaTu-8898s and PaTu-8898t, which are metastatic and non-metastatic, respectively, we showed that the PaTu-8988s cells expressed a lower level of CD9 but had higher proliferation and migration rates than the PaTu-8898t cells. An inverse correlation between CD9 expression and the cell surface level of epidermal growth factor receptor (EGFR) was observed in both cell lines. In the PaTu-8898s cells, overexpression of CD9 decreased the cell surface expression of EGFR, associated with increased expression of dynamin-2, whereas in the PaTu-8898t cells, knockdown of CD9 with RNA interference (RNAi) increased the cell surface expression of EGFR, associated with decreased expression of dynamin-2. However, the total EGFR level did not change by manipulation of CD9 expression, suggesting that $\mathrm{CD} 9$ plays a role in EGFR endocytosis. Furthermore, in the PaTu-8898ts cells, CD9 overexpression decreased the cell proliferation and migration, which were reversed by EGFR overexpression, whereas in the PaTu-8898t cells, CD9 knockdown enhanced the cell proliferation and migration which were blocked by EGFR RNAi both in vitro and in vivo. Thus, in pancreatic cancer cells, downregulation of CD9 may play a role in cancer growth and metastasis through, at least in part, enhancing cell surface expression of EGFR.
\end{abstract}

Correspondence to: Dr Yan Zhao, Department of Gastroenterology, Shanghai Tenth People's Hospital, Tongji University School of Medicine, Shanghai 200072, P.R. China

E-mail: 320zhaoyan@163.com

Key words: pancreatic cancer, CD9, metastasis, epidermal growth factor, endocytosis

\section{Introduction}

Pancreatic cancer is the fourth most common cause of cancer-related mortality in the United States and the eighth worldwide (1). This disease has an extremely poor prognosis with a median survival of 4-12 months and a dismal 5-year survival rate of $5 \%(2,3)$. The lethal nature of pancreatic adenocarcinoma is mainly due to the high frequency of local or regional spread and distant metastasis (4). Although various molecular changes have been revealed in this malignancy, mechanisms underlying the aggressiveness of this neoplasm remain largely unclear.

CD9, a member of the tetraspanin family, is widely expressed on the plasma membrane of various cell types, including hematopoietic cells, endothelial cells, epidermal cells, and many tumor cell lines (5-8). Like other members of the tetraspanins, CD9 interacts with a number of transmembrane proteins, and forms functional complexes, which facilitate cell adhesion, motility and signaling (5,9-14). In pancreatic cancer, CD9 expression is reduced in pancreatic cancer cells but is elevated in the stroma (15) and reduced CD9 expression levels are associated with high tumor grade and lymph node metastasis (16), although contradictory findings have also been reported (17). However, mechanisms underlying the involvement of CD9 in pancreatic cancer metastasis remain incompletely understood.

In the present study, using the two closely associated pancreatic cancer cell lines, PaTu-8988t and PaTu-8988s, which have been shown to be non-metastatic and metastatic in vivo, respectively (18), we demonstrated an inverse correlation between CD9 expression and cell proliferation and motility. PaTu-8988s cells expressed a lower level of CD9 but had higher proliferation and migration rates than the PaTu- $8898 \mathrm{t}$ cells. We further demonstrated an inverse correlation between CD9 level and the cell surface expression of epidermal growth factor receptor (EGFR). CD9 overexpression reduced the cell surface expression of EGFR, associated with increased expression of dynamin-2, whereas CD9 knockdown increased the cell surface expression of EGFR, associated with decreased expression of dynamin-2. While forced expression of EGFR did not reverse CD9 overexpression-mediated inhibition of 
cell proliferation, CD9 knockdown-promoted pancreatic cancer cell proliferation and migration were blocked by EGFR RNAi both in vitro and in vivo. Thus, targeting CD9 may offer therapeutic benefits to patients with pancreatic cancer.

\section{Materials and methods}

Animals. Female severe combined immunodeficient (SCID) mice (4-6 weeks of age) were purchased from Jackson Laboratory. Temperature $\left(20-21^{\circ} \mathrm{C}\right)$ and humidity $(50-60 \%)$ were controlled. Daily light cycle consisted of $12 \mathrm{~h}$ of light and $12 \mathrm{~h}$ of dark. Animals were manipulated under sterile conditions. All animal experiments were carried out after approval by the Institutional Review Board of Shanghai Tenth People's Hospital and were performed in accordance with the Guidelines for the Care and Use of Laboratory Animals published by the National Institutes of Health.

Cell lines. PaTu-8988s and PaTu-8988t cells were obtained from the Cancer Research UK Central Cell Service (Clare Hall Laboratories, Potters Bar, UK). Cells were routinely maintained in Dulbecco's modified Eagle's medium (DMEM) containing $10 \%$ fetal bovine serum (FBS) in a humidified incubator at $37^{\circ} \mathrm{C}$ with an atmosphere of $5 \% \mathrm{CO}_{2}$.

Immunostaining and confocal microscopy. Cells growing on coverslips were fixed with $4 \%$ paraformaldehyde (PFA) for $15 \mathrm{~min}$ at room temperature. They were later blocked with $5 \%$ normal donkey serum containing $0.1 \%$ Triton $\mathrm{X}-100$ in phosphate-buffered saline (PBS) for $1 \mathrm{~h}$ at room temperature. Cells were incubated with an antibody mixture containing a mouse monoclonal anti-CD9 (Abcam) and a rabbit polyclonal anti-EGFR (R\&D Systems) antibody at $4^{\circ} \mathrm{C}$ overnight. After washing 3 times with PBS containing $0.1 \%$ Triton X-100, the cells were incubated with a mixture of Alexa Fluor 488-conjugated donkey anti-mouse IgG (Life Technologies) and Alexa Fluor 594-conjugated donkey anti-rabbit IgG (Life Technologies) for $2 \mathrm{~h}$ at room temperature, and washed 3 times with PBS containing 0.1\% Triton X-100 and incubated with $1 \mu \mathrm{g} / \mathrm{ml} \mathrm{4',6-diamidino-2-phenylindole} \mathrm{(DAPI;} \mathrm{Sigma}$ Aldrich) for $10 \mathrm{~min}$. Confocal microscopy was performed on an LSM-710 laser scanning microscope (Zeiss, Oberkochen, Germany) with a 40x1.3 numerical aperture oil immersion lens. All digital images were captured at the same settings. Final images were processed using Adobe PhotoShop software.

Western blot analysis. Tissues or cells were lysed in $2 \mathrm{X}$ Laemmli buffer containing a cocktail of protease inhibitors (Bio-Rad, Hercules, CA, USA). The proteins were extracted, boiled for $10 \mathrm{~min}$ at $100^{\circ} \mathrm{C}$, and then resolved by SDS-PAGE on a $12 \%$ gel. The protein signals were detected by luminal detection reagent (Santa Cruz Biotechnologies, Santa Cruz, CA, USA). The following primary antibodies were used: anti-CD9 antibody $(1: 1,000)$, anti- $\beta$-actin antibody $(1: 2,000)$, anti-EGFR $(1: 1,000)$, anti-dynamin-2 antibody $(1: 1,000)$ and anti-Na-K-ATPase (1:1,000) (all from Abcam).

Construction of recombinant lentiviral vectors, preparation of lentiviral particles and cell infection. To construct lentiviral
CD9 shRNAs, three oligonucleotides sequences: 5'-GCTGTT CGGATTTAACTTCAT-3' (shCD9-1), 5'-TTCTACACAGGA GTCTATATT-3' (shCD9-2), 5'-CACAAGGATGAGGTGATT AAG-3' (shCD9-3), which specifically target CD9 mRNA (GenBank accession no. NM_001769.3) were synthesized. A scrambled sequence of the CD9 target sequence was used as a negative control. The lentiviral shRNA constructs were prepared at Genomeditech Biotechnology Co. Ltd. (www.genomeditech. com) using the vector U6-MCS-CMV-EGFP. The lentiviral EGFR-shRNA (shEGFR) was constructed similarly using the specific oligonucleotide sequence: 5'-GCAAAGUGUGUA ACGGAAUAG-3') which targets a sequence starting at nucleotide 1247 and lying at the junction of exon 8 and 9 (19). To construct the lentiviral CD9 and EGFR constructs, the CD9 cDNA and EGFR cDNA were amplified by PCR using Pfu polymerase (Promega, USA), and were inserted into the $B a m H I$ and $X h o I$ digestion sites in the lentiviral vector CMV-MCS-IRES-EGFP. Lentiviral particles were prepared at Genomeditech Biotechnology Co. Ltd. Cells were transfected with the lentiviral vectors using Lipofectamine 2000 (Invitrogen Corp, Long Island, NY, USA) according to the manufacturer's instructions.

Cell proliferation assay. PaTu-8898s and PaTu-8898t cells were plated in a 96 -well plate $(2,000$ cells/well $)$, which was incubated for $1-7$ days at $37^{\circ} \mathrm{C}$ in an atmosphere of $5 \% \mathrm{CO}_{2}$ in air. Thereafter, $20 \mu \mathrm{l}$ of CCK- 8 solution was added to each well of the plate and incubated for an additional $4 \mathrm{~h}$ under the same conditions. Then, the absorbance at $450 \mathrm{~nm}$ was measured using a microplate reader, wherein the absorbance value indicated the proliferative capacity.

Scratch-wound assay. The scratch-wound assay was performed as previously described (20). Cells were plated onto 6 -well plates at a concentration of $2 \times 10^{5}$ cells/well. Cell monolayers were carefully wounded by scratching with a sterile plastic pipette tip. The cells were washed twice with PBS and incubated for a further $48 \mathrm{~h}$. Photographs of each wound were captured in the same fields at different times up to $48 \mathrm{~h}$. The distance between the wound edges was analyzed using Image $\mathbf{J}$ version 1.42 (National Institutes of Health, Bethesda, MD, USA). The percentage of wound occupied was calculated by dividing the non-recovered area at $24 \mathrm{~h}$ by the initial wound area at $0 \mathrm{~h}$ and subtracting this value as a percentage from $100 \%$.

In vitro invasion assay. Cells were detached and resuspended in serum-free medium. Cells $\left(4 \times 10^{4}\right.$ cells/well) were then plated into Matrigel-coated invasion chambers (Becton-Dickinson) and allowed to invade for $24 \mathrm{~h}$. The remaining cells in the chambers were removed by Q-tips and the invading cells on the lower surface of the chambers were stained with $0.2 \%$ crystal violet solution. The number of invading cells was calculated by counting three different fields under a phase-contrast microscope and were plotted as the percentage of invading cells of the total number of plated cells.

In vivo tumor growth and metastasis experiments. PaTu-8988t $\left(1 \times 10^{7}\right)$ cells infected with the lentiviral scramble shRNA or CD9-specific shRNA (shRNA3) were subcutaneously 

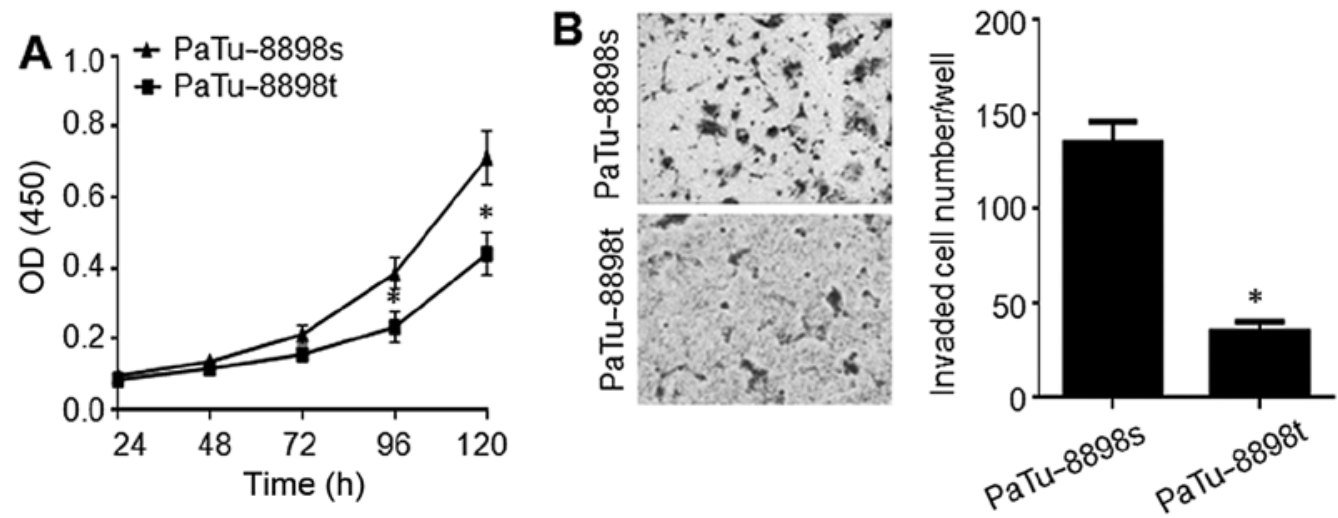

Figure 1. Different proliferation and migration capabilities between the PaTu-8898s and PaTu-8898t cells. (A) Proliferation of the PaTu-8898s and PaTu-8898t cells. Data are the mean \pm SEM from three independent experiments. " $p<0.05$ compared with the PaTu- 8898 s cells. (B) Invasion of the PaTu- $8898 \mathrm{~s}$ and PaTu-8898t cells. Left panel: representative images of the invasion of the PaTu-8898s and PaTu-8898t cells. Right panel: quantification of the invasion of the PaTu-8898s and PaTu-8898t cells. Data are the mean \pm SEM from three independent experiments. ${ }^{*}<<0.05$ compared with the PaTu- $8898 \mathrm{~s}$ cells.

implanted into one flank of the SCID mice ( $\mathrm{n}=12$, respectively). The presence of tumors and their size (length $\mathrm{x}$ width ${ }^{2} \mathrm{x} \pi / 6$ ) were monitored twice weekly. When the animals died or were sacrificed due to tumor burden, the tumors, draining lymph nodes, liver and lungs were harvested and then subjected to histopathological examination. PaTu-8988s $\left(1 \times 10^{6}\right)$ cells infected with the lentiviral CD9 construct or the lentiviral vector were intravenously injected into the tail vein of SCID mice ( $n=12$, respectively). At 120 days after injection, the mice were sacrificed and lungs and livers were collected for $\mathrm{H} \& \mathrm{E}$ staining. Three cross-sections of each lung at different levels (every $100 \mathrm{~mm}$ ) were investigated for assessment of micrometastasis. The percentage of mice with lung metastases and the total number of micrometastatic nodules from the three sections are presented.

Statistical analysis. For the in vitro experiments, the values are expressed as the mean \pm SEM. Comparison between two groups was carried out using the Student's t-test. All experiments were repeated three times. For the in vivo studies, tumor volumes were calculated as the mean \pm SEM. Wilcoxon rank sum test and Kruskal-Wallis test were used to compare treatment differences.

\section{Results}

Different proliferation and migration capabilities between the PaTu-8898s and PaTu-8898t cells. Although PaTu-8898s and PaTu-8898t, two cell lines originating from liver metastases of the same human pancreatic adenocarcinoma, have been shown to be metastatic and non-metastatic in vivo, respectively (18), whether they behave differentially in vitro has rarely been investigated. The proliferation and invasion of both cell lines were investigated by conducting in vitro proliferation and Matrigel invasion assays, respectively. As shown in Fig. 1, PaTu-8898s cells underwent a significantly higher rate of proliferation and invasion compared to the Patu-8898t cells.

Inverse correlation between $C D 9$ expression and cell surface expression of EGFR in the PaTu-8898s and PaTu-8898t cells. To examine the expression of CD9 in both cell lines, the membrane fraction and whole cell lysate were subjected to western blot analysis. As shown in Fig. 2A, the PaTu-8898s cells expressed a markedly lower level of CD9 in both the membrane fraction and the whole cell lysate than did the PaTu-8898t cells. We also examined the expression of EGFR, whose aberrant expression is linked to the etiology of several human epidermal cancers, including pancreatic cancer (21). Intriguingly, a markedly higher level of EGFR was observed in the membrane fraction of the PaTu-8898s than that in the PaTu-8898t cells, while the EGFR level in the whole cell lysate did not differ between the two cell lines (Fig. 2A). The inverse correlation between CD9 and EGFR cell surface expression was confirmed with confocal microscopy. In the PaTu-8898t cells in which CD9 was highly expressed on the cell surface, a large proportion of EGFR was localized in the cytosol (Fig. 2B, arrows), whereas in the PaTu-8898s cells in which a low level of CD9 was observed on the cell surface, EGFR was predominantly localized on the cell surface (Fig. 2B).

CD9 plays a role in EGFR endocytosis. To examine whether CD9 plays a role in the cell surface expression of EGFR, we first examined the effect of CD9 knockdown on the cell surface expression of EGFR. PaTu-8898t cells were infected with lentiviral scramble shRNA (shCT) or CD9 shRNA1-3 (shCD1-3), and the whole cell lysate and membrane fraction were subjected to western blot analysis. Compared to the lentiviral scramble shRNA (shCT)-infected cells, the cells infected with either of the lentiviral shCD9s resulted in a robust downregulation of CD9. Intriguingly, CD9 knockdown did not change the total EGFR expression level in the whole cell lysate but robustly increased the expression of EGFR in the membrane fraction. This was associated with a marked decrease in the expression of dynamin-2, which is known to regulate EGFR endocytosis $(22,23)$, in both the whole cell lysate and membrane fraction (Fig. 3A). Next, we examined the effect of CD9 overexpression on the cell surface expression of EGFR in the PaTu-8898s cells. As shown in Fig. 3B, CD9 overexpression did not change the total EGFR expression level in the whole cell lysate but robustly decreased the expression of EGFR in the membrane fraction, associated with a marked increase in the expression of dynamin- 2 in both the whole cell 
A Membrane fraction

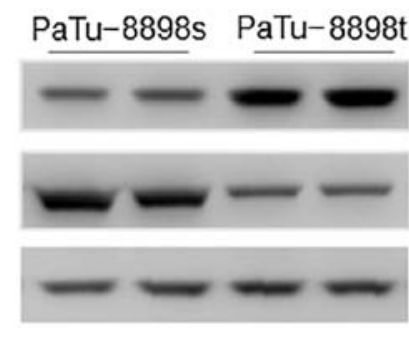

\section{Whole cell lysate}

PaTu-8898s PaTu-8898t

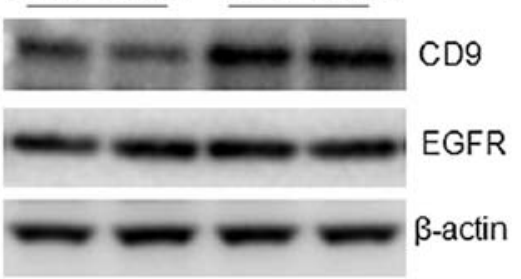

B
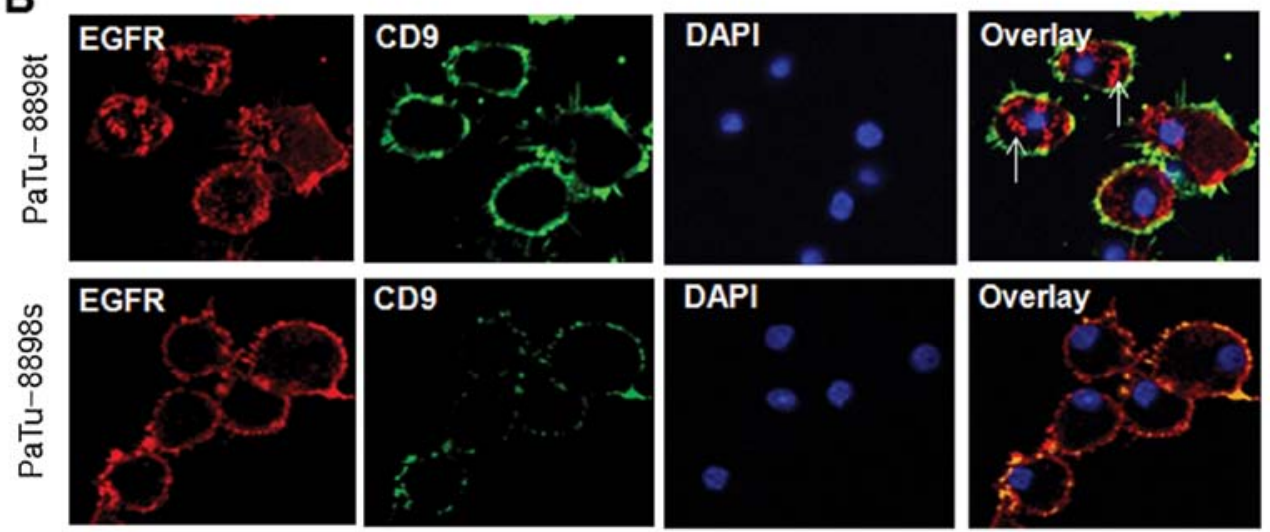

Figure 2. Differential expression of CD9 and EGFR in the PaTu-8898s and PaTu-8898t cells. (A) Western blot analysis of CD9 and EGFR in the whole cell lysate and membrane fraction of the PaTu-8898s and PaTu-8898t cells. Shown are representatives of three independent experiments with similar results. (B) Confocal images showing the subcellular localization of CD9 and EGFR in the PaTu-8898s and PaTu-8898t cells. Shown are representatives of three independent experiments with similar results.
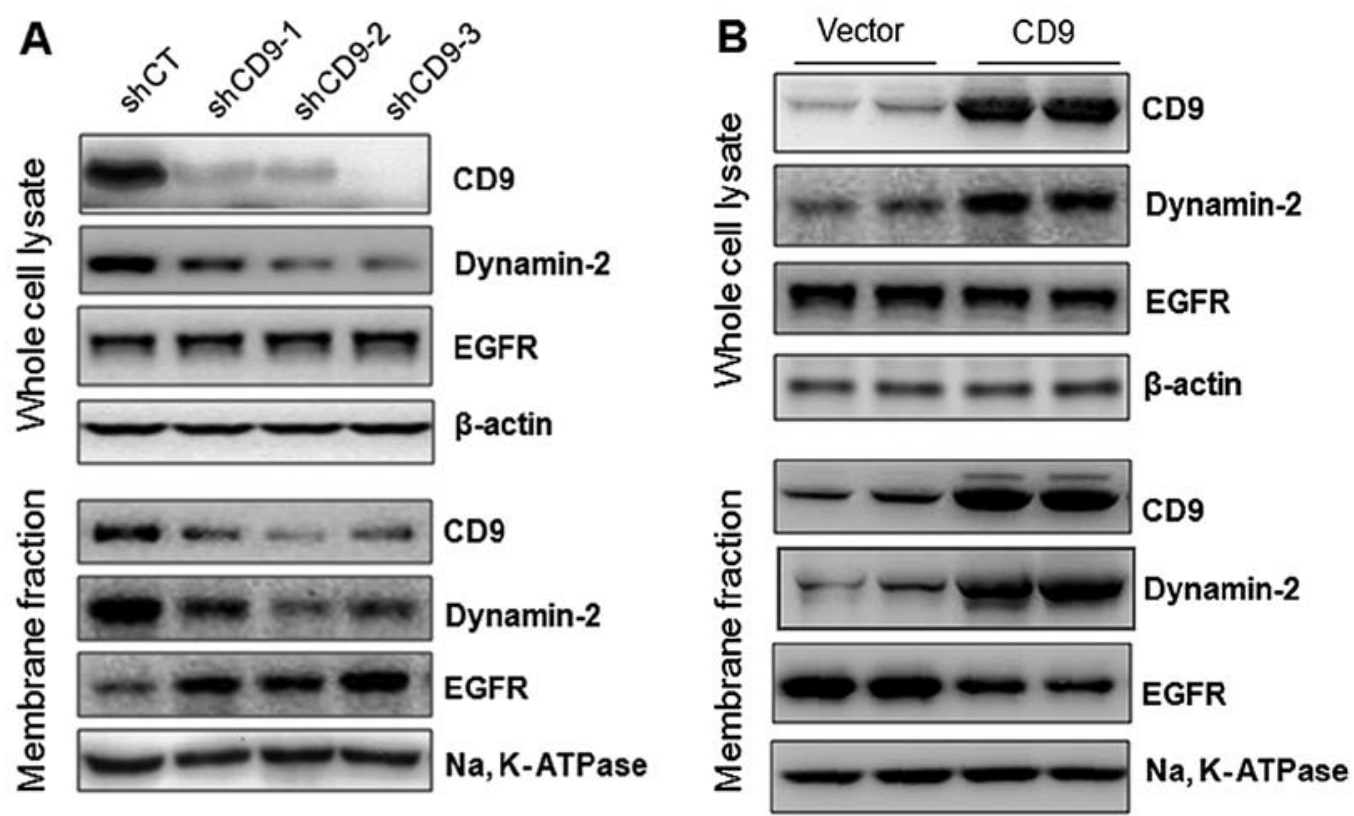

Figure 3. Effect of CD9 knockdown or overexpression on the expression of dynamin-2 and EGFR in the PaTu-8898s and PaTu-8898t cells. (A) Western blot analysis of CD9, dynamin-2 and EGFR in the whole cell lysate and membrane fraction of the PaTu-8898t cells infected with the lentiviral scramble shRNA (shCT), CD9 shRNA-1 (shCD9-1), shCD9-2, or shCD9-3. Shown are representatives of three independent experiments with similar results. (B) Western blot analysis of CD9, dynamin-2 and EGFR in the whole cell lysate and membrane fraction of the PaTu-8898s cells infected with the lentiviral vector or CD9 construct. Shown are representatives of three independent experiments with similar results.

lysate and membrane fraction. The effect of CD9 on EGFR cell surface expression was further visualized with FACS analysis. As shown in Fig. 4, CD9 knockdown in the PaTu-8898t cells resulted in a marked increase in the expression of EGFR on the cell surface. In contrast, CD9 overexpression decreased the cell surface expression of EGFR. 


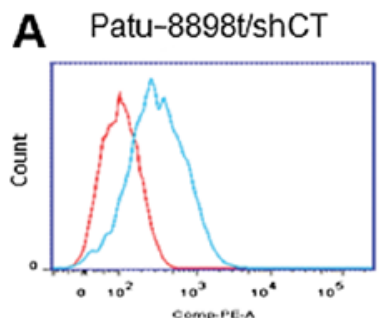

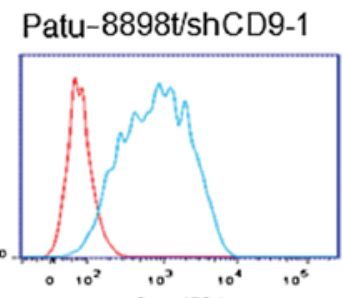

Patu-8898t/shCD9-3

B Patu-8898s/vector

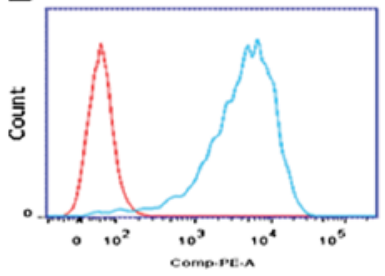

Patu-8898s/CD9

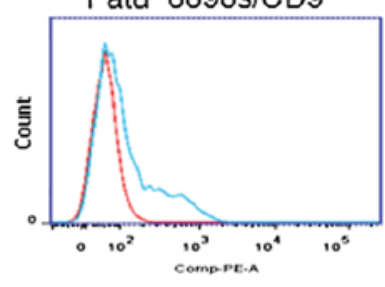

Figure 4. Effect of CD9 knockdown or overexpression on the cell surface expression of EGFR in the PaTu-8898s or PaTu-8898t cells. (A) FACS analysis showing the expression of EGFR (light blue line) on the surface of the PaTu-8898t cells infected with the lentiviral scramble shRNA (shCT), CD9 shRNA-1 (shCD9-1), shCD9-2, or shCD9-3. Shown are representatives of three independent experiments with similar results. (B) FACS analysis of the PaTu-8898s cells infected with the lentiviral vector or CD9 construct using an EGFR-specific antibody (light blue line) vs. the isotype control (red line). Shown are representatives of three independent experiments with similar results.

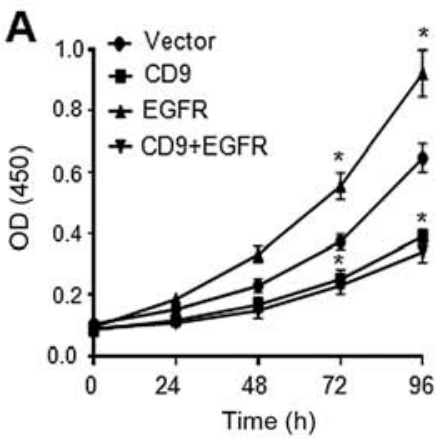

B

$\mathrm{Oh}$

$24 \mathrm{~h}$
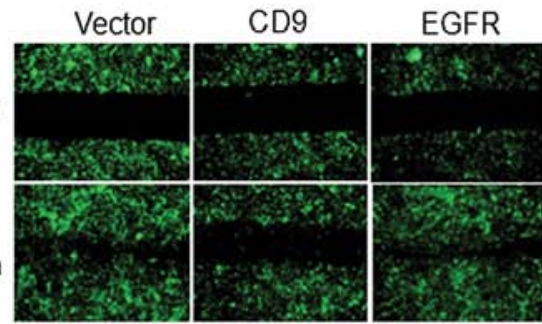

D

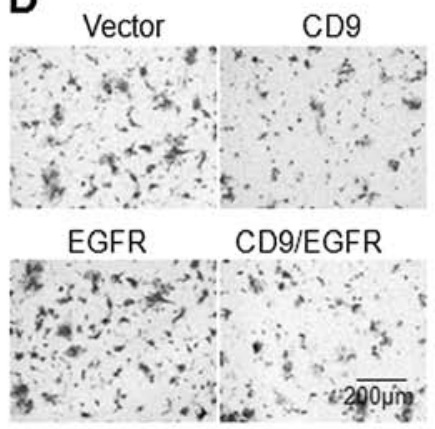

E

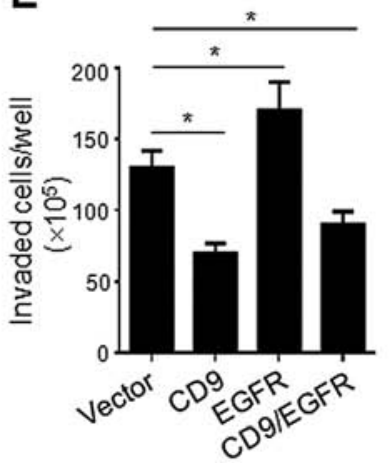

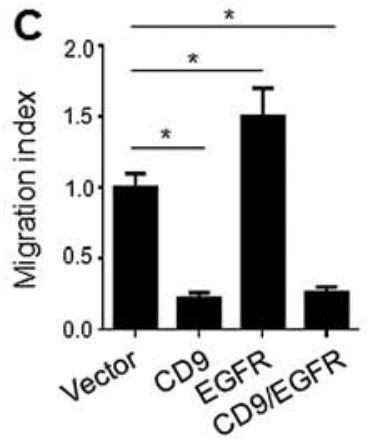

$\mathbf{F}$
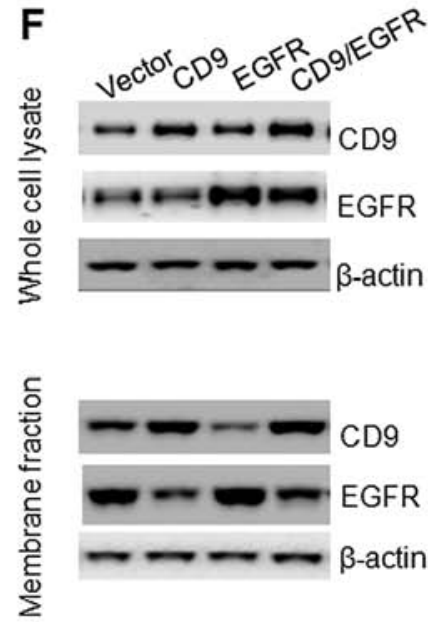

Figure 5. Effect of CD9 overexpression on the proliferation and migration of the PaTu-8898s cells. (A) Proliferation of the PaTu-8898s cells infected with the lentiviral vector, CD9 construct, EGFR construct, or a combination of CD9 and EGFR constructs (1:1 ratio). Data are the mean \pm SEM from three independent experiments. " $\mathrm{p}<0.05$ compared with the PaTu-8898s cells infected with the lentiviral vector. (B) Scratch-wound assay showing the migration of the PaTu-8898s cells infected with the lentiviral vector, CD9 construct, EGFR construct, or a combination of CD9 and EGFR constructs (1:1 ratio). Shown are representatives of three independent experiments with similar results. (C) Quantification of the migration of the PaTu-8898s cells infected with the lentiviral vector, CD9 construct, EGFR construct, or a combination of CD9 and EGFR constructs (1:1 ratio). Data are the mean \pm SEM from three independent experiments. "p $<0.05$ compared with the PaTu-8898s cells infected with the lentiviral vector. (D) Invasion of the PaTu-8898s cells infected with the lentiviral vector, CD9 construct, EGFR construct or a combination of CD9 and EGFR constructs (1:1 ratio) in Matrigel-coated invasion chambers. Shown are representatives of three independent experiments with similar results. (E) Quantification of the invasion of the PaTu-8898s cells infected with the lentiviral vector, CD9 construct, EGFR construct, or a combination of CD9 and EGFR constructs (1:1 ratio). Data are the mean \pm SEM from three independent experiments. "p $<0.05$ compared with the PaTu-8898s cells infected with the lentiviral vector. (F) Western blot analysis of CD9 and EGFR in the whole cell lysate and membrane fraction of the PaTu-8898s cells infected with the lentiviral vector, CD9 construct, EGFR construct, or a combination of CD9 and EGFR constructs (1:1 ratio). Shown are representatives of three independent experiments with similar results. 

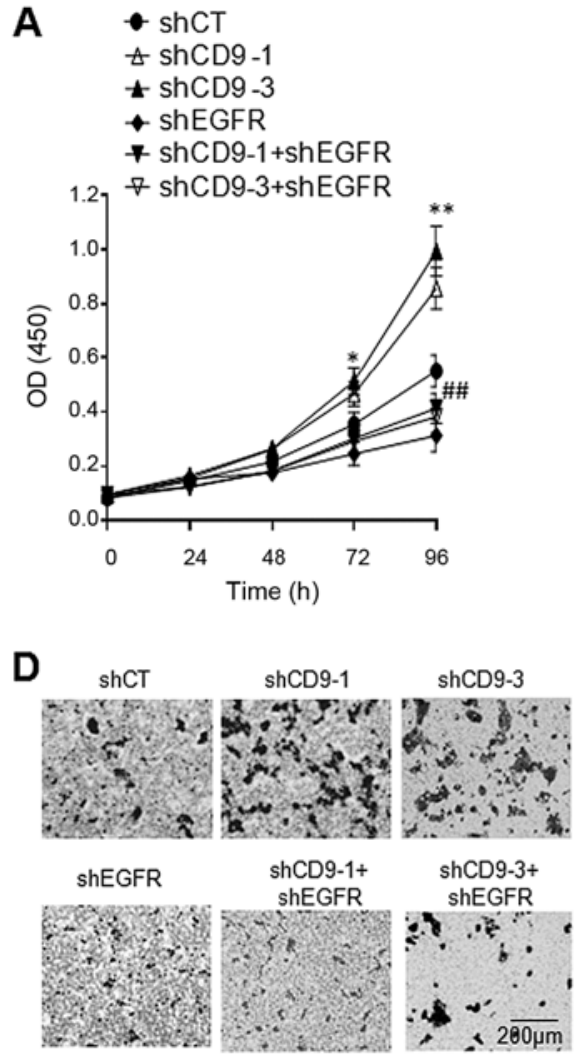
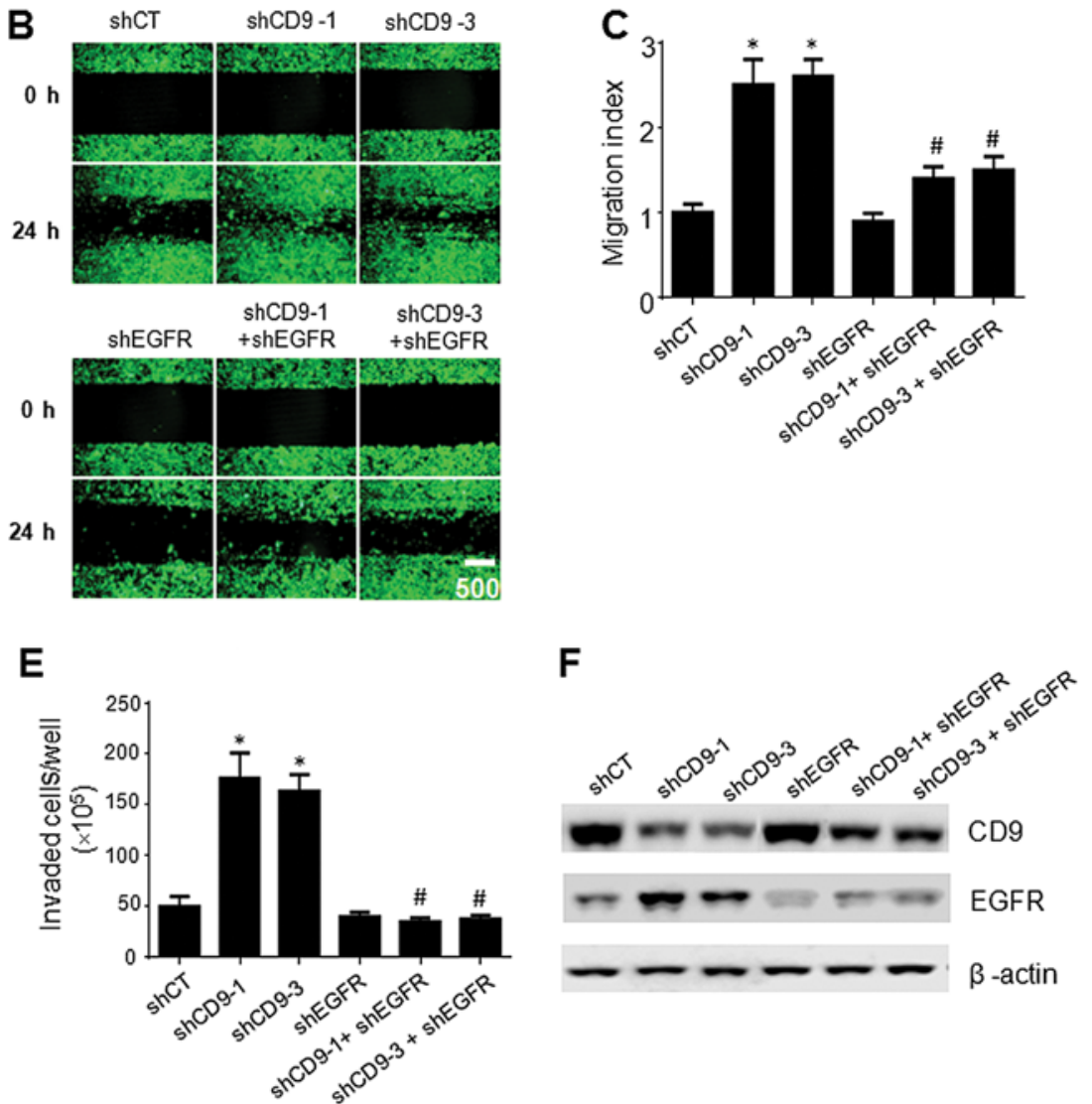

Figure 6. Effect of CD9 knockdown on the proliferation and migration of the PaTu-8898t cells. (A) Proliferation of the PaTu-8898t cells infected with the lentiviral shCT, shCD9-1, shCD9-3, shEGFR, shCD9-1 + shEGFR (1:1 ratio), or shCD9-3 + shEGFR (1:1 ratio). Data are the mean \pm SEM from three independent experiments. ${ }^{*}$ p $<0.01$ compared with the PaTu-8898s cells infected with the lentiviral shCT; ${ }^{\# \#}$ p $<0.01$ compared with the PaTu-8898s cells infected with the lentiviral shCD9-1 or shCD9-3 alone. (B) Scratch-wound assay showing the migration of the PaTu-8898t cells infected with the lentiviral shCT, shCD9-1, shCD9-3, shEGFR, shCD9-1 + shEGFR (1:1 ratio), or shCD9-3 + shEGFR (1:1 ratio). Shown are representatives of three independent experiments with similar results. (C) Quantification of the migration of the PaTu-8898t cells infected with the lentiviral shCT, shCD9-1, shCD9-3, shEGFR, shCD9-1 + shEGFR (1:1 ratio), or shCD9-3 + shEGFR (1:1 ratio). Data are the mean \pm SEM from three independent experiments. * $<<0.05$ compared with the PaTu-8898s cells infected with the lentiviral shCT; ${ }^{*} \mathrm{p}<0.05$ compared with the PaTu-8898s cells infected with the lentiviral shCD9-1 or shCD9-3 alone. (D) Invasion of the PaTu-8898t cells infected with the lentiviral shCT, shCD9-1, shCD9-3, shEGFR, shCD9-1 + shEGFR (1:1 ratio), or shCD9-3 + shEGFR (1:1 ratio) in the Matrigel-coated invasion chambers. Shown are representatives of three independent experiments with similar results. (E) Quantification of the invasion of the PaTu-8898s cells infected with the lentiviral shCT, shCD9-1, shCD9-3, shEGFR, shCD9-1 + shEGFR (1:1 ratio), or shCD9-3 + shEGFR (1:1 ratio). Data are the mean \pm SEM from three independent experiments. ${ }^{*}$ p $<0.05$ compared with the PaTu- 8898 s cells infected with the lentiviral shCT; ${ }^{*}$ p $<0.05$ compared with the PaTu-8898s cells infected with the lentiviral shCD9-1 or shCD9-3 alone. (F) Western blots analysis of CD9 and EGFR in the whole cell lysate and membrane fraction of the PaTu-8898s cells. p<0.05 compared with the PaTu-8898s cells infected with the lentiviral shCT; ${ }^{\#}$ p $<0.05$ compared with the PaTu- 8898 s cells infected with the lentiviral shCD9-1 or shCD9-3 alone. Shown are representatives of three independent experiments with similar results.

CD9 overexpression in the PaTu-8898s cells attenuates cell proliferation, migration and invasion, which is not reversed by EGFR overexpression. To further address the role of CD9 in pancreatic cell proliferation, the PaTu-8988s cells infected with the lentiviral vector, CD9 construct, EGFR construct, or a combination of CD9 and EGFR constructs (1:1 ratio) were subjected to proliferation assay. As shown in Fig. 5A, CD9 overexpression significantly reduced the cell proliferation rate, whereas EGFR overexpression enhanced the cell proliferation. Intriguingly, the CD9 overexpression-mediated inhibition of cell proliferation was not reversed by co-overexpression of EGFR. To examine the effect of CD9 and/or EGFR overexpression on cell migration, PaTu-8988s cells infected with the lentiviral vector, CD9 construct, EGFR construct, or a combination of CD9 and EGFR constructs (1:1 ratio) were subjected to scratch-wound and invasion assays. While EGFR overexpression enhanced cell migration and invasion, CD9 overexpression significantly reduced the cell migration and invasion, which were not reversed by co-overexpression of EGFR. Consistently, western blot analysis showed that while CD9 overexpression reduced the cell surface expression of EGFR, co-overexpression of CD9 and EGFR did not result in any significant increase in EGFR expression on the cell surface (Fig. 5F).

CD9 knockdown promotes pancreatic cancer cell proliferation, migration and invasion, which are reversed by EGFR $R N A i$. We also examined the effect of CD9 knockdown on the proliferation, migration and invasion of PaTu-8898t cells. PaTu$8988 \mathrm{t}$ cells infected with the lentiviral shCD9-1 or shCD9-3 exhibited significantly increased proliferation (Fig. 6A), migration (Fig. 6B and C) and invasion (Fig. 6D and E) compared to the cells infected with the lentiviral shCT, whereas the PaTu-8988t cells infected with the lentiviral shEGFR exhibited significantly decreased proliferation (Fig. 6A), migration (Fig. 6B and C) and invasion (Fig. 6D and E) compared to the cells infected 

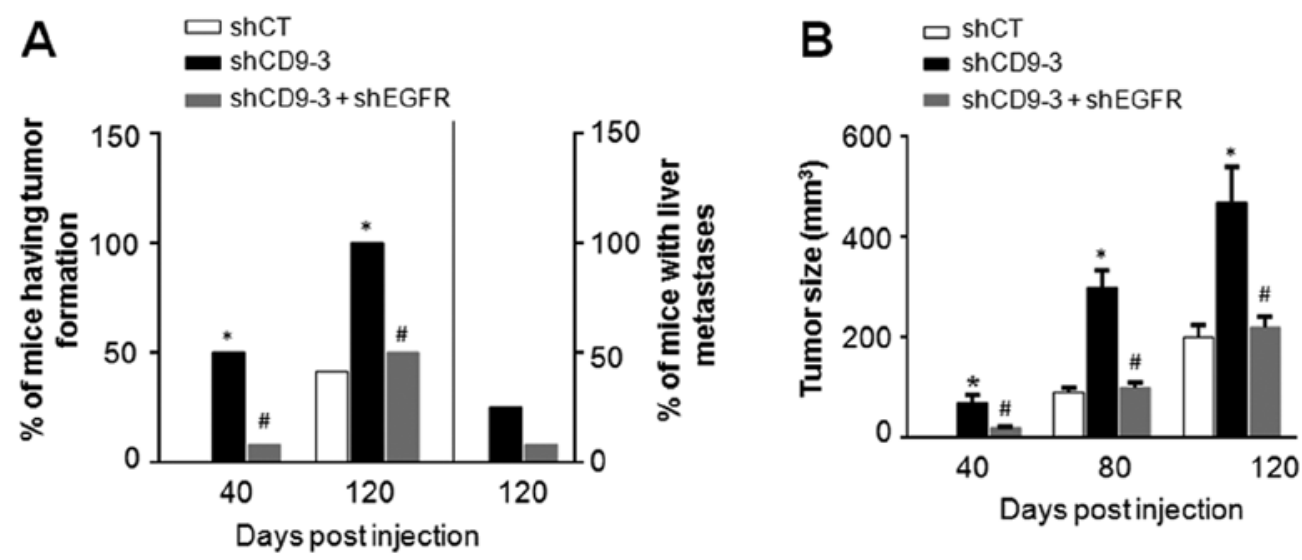

Figure 7. Effect of CD9 and/or EGFR knockdown on tumor growth or metastases in nude mice. PaTu-8988t (1x107) cells infected with the lentiviral shCT, shCD9-3, or shCD9-3 + shEGFR (1:1 ratio) were subcutaneously implanted into one flank of 4- to 6-week-old SCID female mice (n=12/group). (A) Left panel: percentage of tumor formation in the mice 40 and 120 days after injection with the different cells indicated. Right panel: percentage of liver metastases in the mice 120 days after injection with the different cells as indicated. " $p<0.05$ compared with the mice injected with the PaTu- 8898 s cells infected with the lentiviral shCT; ${ }^{*} \mathrm{p}<0.05$ compared with the mice injected with the PaTu-8898s cells infected with the lentiviral shCD9-3 alone. (B) The size of tumors in mice 40, 80 and 120 days after injection with the different cells as indicated. " $\mathrm{p}<0.05$ compared with the mice injected with the PaTu- $8898 \mathrm{~s}$ cells infected with the lentiviral shCT; ${ }^{\#} \mathrm{p}<0.05$ compared with the mice injected with the PaTu-8898s cells infected with the lentiviral shCD9-3 alone.

with the lentiviral shCT. Intriguingly, the co-infection of the cells with the lentiviral EGFR with the lentiviral shCD9-1 or shCD9-3 reversed the increases in cell proliferation, migration and invasion compared to the cells infected with the lentiviral CD9-shRNA1 or lentiviral CD9-shRNA3 alone (Fig. 6A-E). Western blot analysis indicated that co-infection of the cells with lentiviral EGFR shRNA with either lentiviral CD9-shRNA1 or lentiviral CD9-shRNA3 resulted in a marked decrease in EGFR expression on the cell surface compared to the cells infected with the lentiviral CD9-shRNA1 or lentiviral CD9-shRNA3 alone (Fig. 6F).

CD9 knockdown promotes tumor growth and metastasis in vivo. To examine whether $\mathrm{CD} 9$ knockdown affects pancreatic cancer growth and metastasis in vivo, $\mathrm{PaTu}-8988 \mathrm{t}$ cells infected with the lentiviral shCT, shCD9-3, or a combination of lentiviral shCD9-3 and shEGFR (1:1 ratio) were subcutaneously implanted into the right flank of SCID mice. Among the animals injected with the PaTu-8988t cells infected with the lentiviral shCD9-3, 50\% (6/12) of the mice had tumor formation on day 40 , and $100 \%$ (12/12) of mice had tumor formation on day 120. However, among the animals injected with the PaTu-8988t cells infected with a combination of the lentiviral shCD9-3 and shEGFR, only $8 \%(1 / 12)$ of mice had tumor formation on day 40 , and $50 \%$ (6/12) of mice had tumor formation on day 120. In contrast, among the animals injected with the PaTu-8988t cells infected with the lentiviral shCT, no mice had tumor formation on day 40 , and only $41.6 \%(5 / 12)$ of mice had tumor formation on day 120 (Fig. 7A). On day 120, three mice bearing PaTu-8988t cells infected with the lentiviral shCD9-3 had liver metastasis, one mouse bearing PaTu-8988t cells infected with a mixture of lentiviral shCD9-3 and lentiviral shEGFR had liver metastasis, whereas none of the mice bearing PaTu-8988t cells infected with the lentiviral shCT had metastasis (Fig. 7A). At all of the time points, a significantly increased tumor volume was observed in animals bearing the PaTu-8988t cells infected with the lentiviral shCD9-3 compared to those bearing the PaTu-8988t cells infected with the lentiviral shCT (Fig. 7B). However, a marked decrease in tumor volume was observed in the animals bearing the PaTu8988t cells infected with a mixture of lentiviral shCD9-3 and lentiviral shEGFR compared to those bearing the PaTu-8988t cells infected with the lentiviral shCD9-3 alone (Fig. 7B).

\section{Discussion}

Echoing the previous finding that reduced CD9 expression in pancreatic cancer is associated with high tumor grade and lymph node metastasis (16), we demonstrated an inverse correlation between the CD9 expression level and pancreatic cancer cell proliferation and migration using the two closely related pancreatic cancer cell lines, PaTu-8898s and PaTu-8898t. We further demonstrated an inverse correlation between the CD9 level and the cell surface expression of EGFR. CD9 overexpression reduced the cell surface expression of EGFR, associated with increased expression of dynamin-2, whereas CD9 knockdown increased the cell surface expression of EGFR, associated with decreased expression of dynamin-2. While forced expression of EGFR did not reverse CD9-overexpression-mediated inhibition of cell proliferation, CD9 knockdown-promoted pancreatic cancer cell proliferation and migration were blocked by EGFR RNAi both in vitro and in vivo.

Mounting evidence indicates that CD9 negatively regulates cell motility in various cancer cell types (24-26), and CD9 knockout has been shown to increase spontaneous metastasis (27). However, direct evidence for a negative role of CD9 in pancreatic cancer motility has been lacking until the present study. Here, we demonstrated that CD9 overexpression reduced cell motility, whereas its knockdown promoted pancreatic cancer cell motility in vitro and enhanced pancreatic cancer metastasis in vivo. In contrast to the well-established role of CD9 in metastasis, very little is known concerning the involvement of this tetraspanin in the process of cancer cell proliferation. Here, we showed that CD9 knockdown promoted pancreatic cancer cell proliferation, whereas its overexpression 
inhibited cell proliferation. Our data are in line with the previous finding in other cancer cells, such as colon carcinoma cells (28) and small cell lung cancer cells (29). However, controversial results have also been reported. For instance, in lymphoma cell lines, introduction of CD9 expression resulted in significantly increased cell proliferation (30). This discrepancy is not surprising, as tetraspanins have recently been postulated as both cancer suppressors and promoters. This late appreciation is probably due to their capacity to associate with various molecules, which they recruit into special membrane microdomains, and their abundant presence in tumor-derived small vesicles that aid intercellular communication (31).

It is likely that CD9 plays a negative role in pancreatic cancer proliferation and migration through, at least in part, modulation of cell surface expression of EGFR. This notion is based on the findings that i) there was an inverse correlation noted between the CD9 level and cell surface expression of EGFR in pancreatic cancer cells; ii) forced expression of CD9 reduced the cell surface expression of EGFR, whereas knockdown of CD9 promoted EGFR expression on the cell surface; and iii) the CD9 knockdown-mediated increase in pancreatic cancer cell proliferation and migration were blocked by EGFR RNAi. EGFR activation via ligand binding results in signaling through various pathways ultimately resulting in cellular proliferation, survival, angiogenesis, invasion and metastasis. EGFR overexpression is detected in up to $90 \%$ of pancreatic tumors (32), and aberrant expression or activity of EGFR has been strongly linked to the etiology of many other cancers including but not limited to head and neck squamous cell carcinoma (HNSCC), non-small cell lung cancer (NSCLC), colorectal cancer (CRC), breast cancer, pancreatic cancer and brain cancer (21). In line with our findings, previous studies have shown that a decrease inCD9 and an increase in EGFR predict malignant progression of cancer (33), that CD9 associates with EGFR in hepatocellular carcinoma cells and Chinese hamster ovary cancer cells transfected with EGFR/CD9, and that expression of CD9 specifically attenuated EGFR signaling through downregulation of surface expression of EGFR (34).

Based on our findings that CD9 knockdown did not alter EGFR expression in total but rather increased its cell surface expression, we propose that CD9 may play a role in EGFR endocytosis. In support of this hypothesis, we showed that CD9 knockdown resulted in marked decrease in dynamin-2 expression, whereas CD9 overexpression increased dynamin-2 expression. Dynamin, a large GTPase that deforms lipid bilayers, is well known to be involved in the endocytosis of EGFR (22). A recent study has shown that dynamin-2 depletion leads to a strong inhibition of EGFR endocytosis, robust enhancement of EGFR autophosphorylation and ubiquitination, and slower kinetics of EGFR degradation, and that dynamin-mediated endocytosis leads to attenuation of EGFR activation and downstream signaling (23). Thus, CD9 may play a role in EGFR endocytosis through modulation of dynamin expression. However, the mechanisms by which CD9 modulates dynamin-2 expression are largely unknown and should be investigated in future studies.

In summary, we demonstrated that CD9 downregulation, which is evident in many cases of pancreatic cancer, promoted pancreatic cancer cell proliferation and migration through at least in part, enhancing the cell surface expression of EGFR, a well-established target for pancreatic cancer therapy. CD9 played a role in EGFR endocytosis likely through regulating the expression of dynamin, which is known to regulate EGFR endocytosis, although the underlying mechanisms remain to be investigated. These findings lead to the hypothesis that targeting CD9 may offer therapeutic benefits to pancreatic cancer patients.

\section{Acknowledgements}

This study was supported by grants from the Shanghai Science and Technology Commission (no. 11JC1410000), Fund of the Shanghai Health Bureau (no. 20114315) and Training Plan of Excellent Academic Researcher of Shanghai Tenth People's Hospital (nos. 12XSGG105 and 04.01.13037). We appreciate Dr Rui Li from Genomeditech Co. Ltd. (www.genomeditech. com) for the generation of the lentiviral particles.

\section{References}

1. Hezel AF, Kimmelman AC, Stanger BZ, Bardeesy N and Depinho RA: Genetics and biology of pancreatic ductal adenocarcinoma. Genes Dev 20: 1218-1249, 2006.

2. Wray CJ, Ahmad SA, Matthews JB and Lowy AM: Surgery for pancreatic cancer: Recent controversies and current practice. Gastroenterology 128: 1626-1641, 2005.

3. Jemal A, Siegel R, Ward E, Hao Y, Xu J and Thun MJ: Cancer statistics, 2009. CA Cancer J Clin 59: 225-249, 2009.

4. Breslin TM, Hess KR, Harbison DB, Jean ME, Cleary KR, Dackiw AP, Wolff RA, Abbruzzese JL, Janjan NA, Crane CH, et al: Neoadjuvant chemoradiotherapy for adenocarcinoma of the pancreas: Treatment variables and survival duration. Ann Surg Oncol 8: 123-132, 2001.

5. Maecker HT, Todd SC and Levy S: The tetraspanin superfamily: Molecular facilitators. FASEB J 11: 428-442, 1997.

6. Berditchevski F: Complexes of tetraspanins with integrins: More than meets the eye. J Cell Sci 114: 4143-4151, 2001.

7. Boucheix C and Rubinstein E: Tetraspanins. Cell Mol Life Sci 58: 1189-1205, 2001

8. Hemler ME: Tetraspanin proteins mediate cellular penetration, invasion, and fusion events and define a novel type of membrane microdomain. Annu Rev Cell Dev Biol 19: 397-422, 2003.

9. Shaw AR, Domanska A, Mak A, Gilchrist A, Dobler K, Visser L, Poppema S, Fliegel L, Letarte M and Willett BJ: Ectopic expression of human and feline CD9 in a human B cell line confers beta 1 integrin-dependent motility on fibronectin and laminin substrates and enhanced tyrosine phosphorylation. J Biol Chem 270: 24092-24099, 1995.

10. Jones PH, Bishop LA and Watt FM: Functional significance of CD9 association with beta 1 integrins in human epidermal keratinocytes. Cell Adhes Commun 4: 297-305, 1996.

11. Inui $\mathrm{S}$, Higashiyama $\mathrm{S}$, Hashimoto $\mathrm{K}$, Higashiyama $\mathrm{M}$, Yoshikawa $\mathrm{K}$ and Taniguchi $\mathrm{N}$ : Possible role of coexpression of CD9 with membrane-anchored heparin-binding EGF-like growth factor and amphiregulin in cultured human keratinocyte growth. J Cell Physiol 171: 291-298, 1997.

12. Berditchevski F and Odintsova E: Characterization of integrintetraspanin adhesion complexes: Role of tetraspanins in integrin signaling. J Cell Biol 146: 477-492, 1999.

13. Baudoux B, Castanares-Zapatero D, Leclercq-Smekens M, Berna N and Poumay Y: The tetraspanin CD9 associates with the integrin alpha6beta4 in cultured human epidermal keratinocytes and is involved in cell motility. Eur J Cell Biol 79: 41-51, 2000.

14. Shi W, Fan H, Shum L and Derynck R: The tetraspanin CD9 associates with transmembrane TGF-alpha and regulates TGF-alpha-induced EGF receptor activation and cell proliferation. J Cell Biol 148: 591-602, 2000.

15. Crnogorac-Jurcevic T, Efthimiou E, Capelli P, Blaveri E, Baron A, Terris B, Jones M, Tyson K, Bassi C, Scarpa A, et al: Gene expression profiles of pancreatic cancer and stromal desmoplasia. Oncogene 20: 7437-7446, 2001.

16. Sho M, Adachi M, Taki T, Hashida H, Konishi T, Huang CL, Ikeda N, Nakajima Y, Kanehiro H, Hisanaga M, et al: Transmembrane 4 superfamily as a prognostic factor in pancreatic cancer. Int J Cancer 79: 509-516, 1998. 
17. Grønborg M, Kristiansen TZ, Iwahori A, Chang R, Reddy R, Sato N, Molina H, Jensen ON, Hruban RH, Goggins MG, et al: Biomarker discovery from pancreatic cancer secretome using a differential proteomic approach. Mol Cell Proteomics 5: 157-171, 2006.

18. Elsässer HP, Lehr U, Agricola B and Kern HF: Establishment and characterisation of two cell lines with different grade of differentiation derived from one primary human pancreatic adenocarcinoma. Virchows Arch B Cell Pathol Incl Mol Pathol 61: 295-306, 1992.

19. Chen G, Kronenberger P, Teugels E, Umelo IA and De Grève J: Targeting the epidermal growth factor receptor in non-small cell lung cancer cells: The effect of combining RNA interference with tyrosine kinase inhibitors or cetuximab. BMC Med 10: 28, 2012.

20. Liang CC, Park AY and Guan JL: In vitro scratch assay: A convenient and inexpensive method for analysis of cell migration in vitro. Nat Protoc 2: 329-333, 2007.

21. Brand TM, Iida M, Li C and Wheeler DL: The nuclear epidermal growth factor receptor signaling network and its role in cancer. Discov Med 12: 419-432, 2011

22. Sousa LP, Lax I, Shen H, Ferguson SM, De Camilli P and Schlessinger J: Suppression of EGFR endocytosis by dynamin depletion reveals that EGFR signaling occurs primarily at the plasma membrane. Proc Natl Acad Sci USA 109: 4419-4424, 2012.

23. Schroeder B, Weller SG, Chen J, Billadeau D and McNiven MA: A Dyn2-CIN85 complex mediates degradative traffic of the EGFR by regulation of late endosomal budding. EMBO J 29: 3039-3053, 2010.

24. Chen S, Sun Y, Jin Z and Jing X: Functional and biochemical studies of CD9 in fibrosarcoma cell line. Mol Cell Biochem 350: 89-99, 2011.

25. Fan J, Zhu GZ and Niles RM: Expression and function of CD9 in melanoma cells. Mol Carcinog 49: 85-93, 2010.

26. Ono M, Handa K, Withers DA and Hakomori S: Motility inhibition and apoptosis are induced by metastasis-suppressing gene product CD82 and its analogue CD9, with concurrent glycosylation. Cancer Res 59: 2335-2339, 1999.
27. Copeland BT, Bowman MJ, Boucheix C and Ashman LK: Knockout of the tetraspanin Cd9 in the TRAMP model of de novo prostate cancer increases spontaneous metastases in an organ-specific manner. Int J Cancer. 133: 1803-1812, 2013.

28. Ovalle S, Gutiérrez-López MD, Olmo N, Turnay J, Lizarbe MA, Majano P, Molina-Jiménez F, López-Cabrera M, Yáñez-Mó M, Sánchez-Madrid F, et al: The tetraspanin CD9 inhibits the proliferation and tumorigenicity of human colon carcinoma cells. Int J Cancer 121: 2140-2152, 2007.

29. Zheng R, Yano S, Zhang H, Nakataki E, Tachibana I, Kawase I, Hayashi S and Sone S: CD9 overexpression suppressed the liver metastasis and malignant ascites via inhibition of proliferation and motility of small-cell lung cancer cells in NK cell-depleted SCID mice. Oncol Res 15: 365-372, 2005.

30. Herr MJ, Longhurst CM, Baker B, Homayouni R, Speich HE, Kotha $\mathbf{J}$ and Jennings LK: Tetraspanin CD9 modulates human lymphoma cellular proliferation via histone deacetylase activity. Biochem Biophys Res Commun 447: 616-620, 2014.

31. Zöller M: Tetraspanins: Push and pull in suppressing and promoting metastasis. Nat Rev Cancer 9: 40-55, 2009.

32. Troiani T, Martinelli E, Capasso A, Morgillo F, Orditura M, De Vita F and Ciardiello F: Targeting EGFR in pancreatic cancer treatment. Curr Drug Targets 13: 802-810, 2012.

33. Nankivell P, Williams H, McConkey C, Webster K, High A, MacLennan K, Senguven B, Rabbitts P and Mehanna H: Tetraspanins CD9 and CD151, epidermal growth factor receptor and cyclooxygenase-2 expression predict malignant progression in oral epithelial dysplasia. Br J Cancer 109: 2864-2874, 2013.

34. Murayama Y, Shinomura Y, Oritani K, Miyagawa J, Yoshida H, Nishida M, Katsube F, Shiraga M, Miyazaki T, Nakamoto T, et al: The tetraspanin CD9 modulates epidermal growth factor receptor signaling in cancer cells. J Cell Physiol 216: 135-143, 2008. 